\title{
Prácticas diagnósticas y riesgo social: la radicalización de la individualización ${ }^{1}$
}

\author{
Carolina González Laurino* \\ Sandra Leopold Costábile**
}

RESUMEN

Este trabajo analiza críticamente el discurso experto en las prácticas diagnósticas en el ámbito socio-jurídico y en el sistema de protección social a la infancia en el Uruguay, cuya forma de argumentación teórica parece apoyarse en el discurso de la modernidad reflexiva. Desde esta perspectiva, los expertos retoman el lenguaje del riesgo a partir del supuesto de la construcción auto-reflexiva de la biografía de los sujetos.

Palabras clave: Reflexividad / Individualización / Diagnóstico / Riesgo social.

\section{Práticas diagnósticas e risco social: A radicalización da individualización}

Este trabalho analisa criticamente o discurso experiente nas práticas diagnósticas no âmbito sócio-jurídico e no sistema de protecção social à infância no Uruguai, cuja forma de argumentación teórica parece se apoiar no discurso da modernidad reflexiva. Desde esta perspectiva, os experientes retomam a linguagem do risco a partir do suposto da construção autorreflexiva da biografia dos sujeitos.

Palavras chave: Reflexividad / Individualización / Diagnóstico / Risco social.

1 Este trabajo parte de una investigación realizada en el contexto de la edición 2008 de los Fondos Concursables Carlos Filgueira del Programa de Infancia y Familia del Ministerio de Desarrollo Social uruguayo que se orientó al análisis de las prácticas diagnósticas de la institución de asistencia a la infancia y la adolescencia -producidas u ordenadas desde el ámbito judicial-como fuente de conocimiento acerca de las familias uruguayas. A nivel metodológico se tomaron como unidades de análisis los informes diagnósticos elaborados por el cuerpo técnico de los Juzgados de Familia Especializados y de la División de Estudio y Derivación del Instituto de la Niñez y la Adolescencia en Uruguay seleccionados en función de criterios aleatorios de análisis muestral cuya trayectoria se siguió desde el sistema judicial a la institución asistencial.

* Uruguaya. Doctora en Sociología y Ciencias Políticas. Licenciada en Sociología. Asistente Social. Docente e investigadora del Departamento de Trabajo Social. Facultad de Ciencias Sociales. Universidad de la República. Correo electrónico: carolsoc@gmail.com

** Uruguaya. Magíster en Trabajo Social. Asistente Social. Docente e investigadora del Departamento de Trabajo Social. Facultad de Ciencias Sociales. Universidad de la República. Correo electrónico: sleopold@adinet.com.uy. 


\section{Practices diagnósticas and social risk: The radicalización of the individualization}

\section{ABSTRACT}

This work juridical associate analyzes critically the expert speech in diagnostic practices in judicial and social protection system to the infancy in the Uruguay, which form of theoretical argumentation seems to rest on the speech of the reflexive modernity. From this perspective, the experts recapture the language of the risk from the reflexive biographical construction.

Key words: Reflexivity / Individualization / Diagnostic / Social risk. 


\section{Sociedad de riesgo e individualización: la construcción de una biografía reflexiva}

La "modernidad reflexiva", como llama Giddens a los procesos de modernidad "reciente"; la "modernidad tardía", como la adjetiva Habermas, tanto como la "modernidad líquida", en la metáfora de Bauman, o la "modernidad invertida" como la denomina Hobsbawm, constituyen solo algunos intentos de nominar profundos procesos de transformación que aparecen frente al análisis social de aquellos autores contemporáneos que han decidido resistirse a la idea de la posmodernidad como forma de caracterizar un período de la historia de Occidente que pretende dar sepultura a las promesas ilustradas de emancipación humana mediante el proceso de racionalización que construye el proyecto moderno.

Giddens habla de tres elementos para explicar el carácter dinámico de la vida social moderna: (I) la separación entre tiempo y espacio, (II) el desenclave de las instituciones sociales y (III) la reflexividad generalizada (Giddens 1995: 28-34).

No obstante, es la tercera de las características anunciadas por Giddens -el carácter reflexivo de la modernidad- lo que en este momento interesa subrayar. Tal carácter supone, por una parte, una revisión constante de la mayoría de los aspectos de la vida social, como consecuencia de la permanente transformación del conocimiento. Por otra parte, como característica de la modernidad tardía, la reflexividad constituye una inflexión en la forma de concebir los condicionamientos sistémicos o las determinaciones institucionales sobre el sujeto moderno.

Desde esta visión, los actores sociales son capaces de sopesar las opciones y las consecuencias de sus actos, mediante un proceso de análisis que los libera de las fuertes determinaciones institucionales y societales que constreñían sus decisiones. Esta mayor independencia en la búsqueda de herramientas con las que manejar su biografía otorgaría a los actores individuales y colectivos un mayor poder de injerencia en sus asuntos, lo que, a la vez que más libres, los convertiría en responsables de sus propias decisiones. De este modo, con esta capacidad de decisión sobre sus trayectorias personales y sociales los sujetos se enfrentan a los múltiples riesgos que aparecen con la vida moderna. ${ }^{2}$

2 Como explica Giddens, el cálculo de riesgos y oportunidades en la vida del sujeto 
Beck, posicionado al igual que Giddens, desde la perspectiva de la modernidad reflexiva, identifica la aparición de la sociedad de riesgo como resultado de la obsolescencia de la sociedad industrial. Reconoce con este concepto, "una fase de desarrollo de la sociedad moderna en la que los riesgos sociales, políticos, económicos e individuales tienden cada vez más a escapar a las instituciones de control y protección de la sociedad industrial" (Beck 1994:18).

Para Beck, en la sociedad industrial clásica, las formas de vida colectiva se asemejaban a las muñecas rusas que se colocan unas dentro de otras. Esta imagen grafica la forma en que las categorías de las situaciones vitales -clase, familia nuclear, género, división del trabajo y matrimonio- se relacionaban unas a otras.

En la sociedad de riesgo, estas categorías y su modalidad de articulación, están siendo sistemáticamente sometidas a procesos de desvinculación y revinculación. De aquí resulta, en palabras de Beck, el significado de la teoría de la individualización, entendida esta como "una compulsión, pero una compulsión a fabricar, auto-diseñar y auto-escenificar no solo la propia biografía, sino también sus compromisos y redes de relaciones a medida que cambian las preferencias y fases de la vida. (...) Individualización significa que la biografía estándar se convierte en una biografía de elección, una biografía hágalo usted mismo (Ronald Hitzler) o, en expresión de Giddens, una biografía reflexiva." (Beck 1994: 29-30)

En este mismo sentido, Giddens propone la idea de un yo analíticamente construido mediante la "política de la vida" que define como una política de "estilo de vida (...) se trata de una política de realización del yo en un entorno reflejamente ordenado, donde esa reflexividad enlaza el yo y el cuerpo en sistemas de ámbito universal" (Giddens 1995: 271).

En síntesis, desde los riesgos socialmente producidos, los teóricos de la modernidad reflexiva invitan a pensar en la cuota parte que al individuo corresponde desde las políticas de la vida en la gestión de sus propias actitudes y conductas respecto a la eventualidad de distintos tipos de riesgo. Como se exponía anteriormente, el sujeto es visualizado frente a un abanico de

moderno, lo coloca frente a la posibilidad de adoptar una decisión informada y en pleno conocimiento de las consecuencias de su acción como centro de la posibilidad de colonización del futuro. "En la sociedades modernas, cuanto mayores sean los esfuerzos del individuo por forjarse reflejamente una identidad de su yo, tanto más consciente será de que su práctica habitual configura los resultados futuros" (Giddens, 1995:166). 
opciones y al cálculo de sus posibles ganancias y riesgos en la construcción reflexiva de su propia biografía, tanto como sus efectos universales. ${ }^{3}$

Sin embargo, esta perspectiva teórica que habla de un sujeto informado y autoconsciente en la construcción de su propia identidad, trae aparejadas algunas dificultades a la hora de la individualización de los riesgos en poblaciones excluidas, si consideramos el contexto de una sociedad heterogénea y desigual en la que se enmarcan las decisiones de los actores.

\section{De seguridades y riesgos: una perspectiva crítica}

En la descripción de los teóricos de la modernidad reflexiva no aparecen limitaciones a ese proceso de creciente reflexividad individual. No obstante, y pese a sucesivas aclaraciones de Giddens, ${ }^{4}$ la pregunta por el desarrollo autónomo del yo en condiciones sociales desventajosas aparece nuevamente pertinente. ¿Es posible pensar en un sujeto autónomo, comprometido con la decisión de su propia vida en proyección universal -como se plantea en la conceptualización de la política de la vida- cuando se habla de personas excluidas, desafiliadas -en la concepción de Castel- o precariamente incluidas en la vida social, económica, política y cultural de una sociedad nacional?

Plantear esta pregunta supone un doble riesgo. Por un lado, responder afirmativamente posiciona al analista en el lugar de la responsabilización individual de actitudes, comportamientos y representaciones sociales en las que habría que indagar cimientos sociales. Por otro lado, la respuesta negativa a la pregunta corre el riesgo de comprometer al investigador, no solo en la búsqueda mecánica de causas sociales de conductas individuales, sino en la producción de respuestas prefabricadas y de metas externas a los valores y deseos de personas pertenecientes a amplios sectores

3 "Hasta aquí -sostiene Giddens- nuestro análisis nos lleva al mundo de las relaciones sociales externas al yo en función, casi siempre de su impacto reflejo sobre la identidad y el estilo de vida. Sin embargo, las decisiones personales afectan también a consideraciones universales (el nexo, en este caso, va de las personas al planeta)". (Giddens 1995: 279).

4 Esta objeción se encuentra explicitada en la introducción de Modernidad e identidad del yo: “Podría pensarse que con la expresión «estilo de vida» nos referimos solo a los logros de grupos o clases más favorecidas [... ] Los pobres quedarán más o menos excluidos de la posibilidad de realizar elecciones de estilo de vida". Sin embargo, Giddens sostiene que su reflexión abarca a todas las clases sociales, incluyendo contextos de privación social (Giddens 1995:14). 
sociales, con la consiguiente consideración de sus proyectos en situación de "minoridad" social.

No obstante, partir de la conceptualización que supone que todos los individuos tienen intrínsecamente, la misma posibilidad de hacerse cargo de decisiones adoptadas libremente, aun en contextos de pobreza y exclusión social, como si esta situación no condicionara seriamente sus opciones de vida, sería caer en la ingenuidad de ignorar la producción social de las desigualdades en un sistema de clases.

Las opciones individuales, y en este punto puede completarse el análisis de Giddens, son históricamente situadas y contextualizadas en sociedades modernas complejas. Desde otras perspectivas teóricas se observa con preocupación, el carácter deficitario de este proceso de creciente reflexividad individual, enmarcado en una contemporaneidad en la que, al mismo tiempo que se incrementa la generación de riesgos, se fragilizan los soportes colectivos. En este sentido, afirma Castel (2004) que dos series de transformaciones se conjugan y dan sostén a la problemática de la inseguridad. En primer lugar, señala la erosión de los sistemas de producción de seguridad "clásicos", que contrarrestaban los riesgos sociales, en el marco de la sociedad salarial sobre la base de condiciones de trabajos estables. Desde su perspectiva, el debilitamiento del Estado nacional más los cambios socioeconómicos generados desde mediados de los años '70, han producido un estado de inseguridad frente al porvenir, en el que también se ve incrementada la inseguridad civil.

A este debilitamiento de la protección clásica, Castel agrega en segundo término, la irrupción de una nueva generación de riesgos -riesgos industriales, tecnológicos, ecológicos, sanitarios, naturales, etcétera- cuya emergencia resulta del devenir de las ciencias y de las tecnologías que, en su particular desarrollo, parecerían haberse vuelto "contra" la naturaleza y el medio ambiente, generando así un sentimiento generalizado de impotencia.

La paradójica relación entre riesgo y seguridad es claramente dejada en evidencia por Castel (2004), quien no duda en señalar que si consideramos el entramado de protecciones civiles y sociales $^{5}$ que se han dispuesto -por lo pronto en las

5 Robert Castel (2004) distingue dos grandes tipos de protecciones. Las protecciones civiles son las que garantizan las libertades fundamentales y la seguridad de los bienes y 
sociedades de los países desarrollados- seguramente estas sean la sociedades más seguras que jamás hayan existido. Pero, al mismo tiempo, y en forma perturbadora, las preocupaciones en torno a la seguridad se observan omnipresentes. La "frustración sobre la situación de la seguridad", tal como la denomina Castel, se deriva del hecho de que los programas de protección no solo no son absolutamente eficientes, sino que, además, al mismo tiempo que dominan -relativamente- ciertos riesgos, hacen emerger otros nuevos.

¿Es en este sentido que puede comprenderse la afirmación de Castel (2004:13) de que "estar protegido es también estar amenazado"? En términos hipotéticos, plantea que la conjunción de las transformaciones por él enunciadas, "alimenta la frustración acerca de la seguridad", en virtud de que el incremento de la sensibilidad a los riesgos configura una búsqueda infinita y siempre frustrada de la seguridad.

Por otra parte, al igual que Luhmann (1992), Castel distingue riesgo de peligro, pero sus preocupaciones tienen sentidos diversos. Este último, concibe al riesgo como "un acontecimiento previsible, cuyas probabilidades de producirse pueden estimarse, así como el costo de los daños que provocará", a su vez, agrega, es un acontecimiento que "puede ser indemnizado porque puede ser mutualizado" (Castel 2004: 77). Es en este sentido, que el seguro ha constituido, para Castel, "la gran tecnología" que posibilitó el control de los riesgos, de manera tal que los sujetos se involucraron en nucleamientos colectivos cuyos miembros se reparten el costo de los riesgos. Por ello el sostén de la cobertura de los riesgos sociales se configura como un modelo solidario o mutual.

La relevancia de esta distinción introducida por Castel entre riesgo y peligro o entre riesgo y daño, pretende disolver la confusión entre ambos conceptos, en franca controversia con los posicionamientos que vienen incentivando la inflación contemporánea de la noción de riesgo que incrementa las demandas de seguridad y al mismo tiempo disuelve las posibilidades de estar protegido.

Por ello, afirma Castel, la "cultura del riesgo" -tal cual refiere Giddens- fabrica peligro, al igual que cuando Beck (1998) enuncia la sociedad del riesgo está colocando a la incertidumbre y

de las personas en el marco de un Estado de Derecho. Las protecciones sociales "cubren" a los sujetos de los principales riesgos que puedan suponer una degradación de su situación vital: accidentes, enfermedad, vejez, pobreza. 
a la inseguridad como principios rectores del porvenir de la civilización. Por tanto, agrega: “evocar legítimamente el riesgo no consiste en colocar la incertidumbre y el miedo en el corazón del porvenir, sino por el contrario, en tratar de hacer del riesgo un reductor de incertidumbre para dominar el porvenir, desarrollando medios apropiados para hacerlo más seguro" (Beck 2004: 77). En este sentido, vale subrayar nuevamente, que el dominio de los riesgos clásicos supuso su "mutualización", en el marco de la responsabilidad colectiva.

No es extraño, entonces, continúa Castel, que la noción generalizada e indiferenciada del riesgo aportada tanto por Beck como por Giddens, contribuya teóricamente para denunciar el carácter obsoleto de los dispositivos clásicos de protección, precisamente en lo que refiere a su dimensión colectiva. A la vez que se insiste en la proliferación masiva de los riesgos, se efectiviza "una celebración del individuo aislado de sus inserciones colectivas, "desarraigado" (disembedded), según la expresión de Giddens" (Castel, 2004:82).

Se establece, así, una estrecha relación entre la multiplicación de los riesgos, la hiperindividualización de las prácticas y la privatización de los seguros, de manera tal que la protección ante los riesgos pasa a depender de una estrategia individual -el sujeto debe "asegurarse a sí mismo"- a la vez que se asegura el porvenir de los seguros a través de la proliferación de los riesgos. En frontal controversia con este posicionamiento que promueve la privatización de los riesgos, Castel propone clarificar la dimensión social de los nuevos factores de incertidumbre, a la vez que formular interrogantes acerca de las posibilidades de que los mismos puedan ser enfrentados colectivamente. Esta tarea la reconoce como inmensamente difícil en la actualidad, pero a la vez insoslayable, en tanto no le resulta exagerado afirmar que "la necesidad de protección forma parte de la «naturaleza» social del hombre contemporáneo, como si el estado de seguridad se hubiera vuelto una segunda naturaleza, e incluso el estado natural del hombre social" (Castel 2004: 85).

En síntesis, las perspectivas teóricas presentadas subrayan coincidentemente los crecientes procesos de individualización, en el marco de importantes cambios societales que vienen acaeciendo en el mundo occidental desde el último cuarto del siglo XX y que someten a cuestión la vigencia y la viabilidad de las antiguas promesas ilustradas de emancipación humana. 
Pero, mientras los teóricos de la modernidad reflexiva enfatizan que el creciente proceso de individualización -resultado de una mayor reflexividad- libera al sujeto de condicionamientos sistémicos o determinaciones institucionales, habilitándole así una amplia libertad biográfica, otros señalan el carácter deficitario de dicho proceso, reconociendo el desarrollo de un individualismo negativo, como resultado de la fragilización de los soportes colectivos, y que afecta a los sujetos más vulnerables, colocándolos en una perspectiva de atomización, aislamiento y desconexión (SVAMPA, 2000).

En tiempos de individualización creciente, ¿cómo se presentan y comprenden, desde el discurso experto en las prácticas diagnósticas, los sujetos protagonistas de situaciones familiares que ingresan al ámbito socio-jurídico y al sistema de protección social a la infancia en Uruguay?

En páginas anteriores se exponían los fundamentos conceptuales de los teóricos de la modernidad reflexiva, que plantean la conceptualización del riesgo como llave de interpretación de fenómenos que surgen en la modernidad tardía. Teniendo en cuenta las proyecciones emancipatorias de la Ilustración para el individuo moderno al que se le presentan varias opciones -sobre las que deberá tomar una decisión, con sus oportunidades y riesgos-, se lo habilitaría a construir una política de estilo de vida. ¿Es este modelo de hombre moderno, libre de decidir ante una serie de alternativas, quien aparece como supuesto de referencia en los diagnósticos sociales del sistema judicial y del ámbito experto de protección social uruguayo? ¿O por el contrario, se exponen -desde una perspectiva crítica a la individualización creciente- sujetos aislados, carentes de recursos, inmersos en un contexto de fragilización de los soportes colectivos?

Ante este documento que constituye el informe de diagnóstico -al que entendemos como resto arqueológico o monumento en el sentido que le fuera asignado por Michel Foucault- en primer lugar cabría preguntarse desde qué supuestos o referencias teóricas parten los sistemas expertos para hacer la lectura de la biografía del sujeto o grupo familiar. En segundo término, corresponde plantear la interrogante acerca de la historicidad de este modelo o conceptualización de referencia. ¿Se trata de un modelo situado y contextualizado social, cultural, económica y políticamente? 


\section{Diagnóstico y lenguaje de riesgo}

Frente al desafío de la elaboración de una síntesis del escenario social de una familia cuyas afiliaciones al sistema están seriamente socavadas, los expertos resumen la situación como de "riesgo social". Es preciso problematizar esta afirmación para analizar qué supuestos están detrás -o delante- de una manifestación conceptual de estas características. ¿Cuál es el concepto de riesgo que se maneja en estos discursos expertos? ¿A qué remite el "riesgo social"? ¿Qué tipo de respuestas institucionales se esperan frente a este diagnóstico experto?

La idea de "riesgo" es utilizada con sentidos diversos en función de los intereses y objetivos que se persigan en los ámbitos especializados que recurren a este dispositivo conceptual. La probabilidad de que un suceso -generalmente temible o no deseado-ocurra, se presenta mediante un planteo abstracto, que reduce el contenido a una fórmula matemática de cálculo en la que se sopesan indicadores de predisposición. De esta forma, en el empleo de la categoría del riesgo por los sistemas abstractos se revelan sus características de pragmatismo y versatilidad que lo construyen como un dispositivo técnico de alta complejidad para su uso profano.

A través de estos complejos mecanismos se elabora una idea de riesgo aparentemente neutral en sus connotaciones, que se presenta mediante un discurso tecnocrático despolitizado que es preciso desmontar para descubrir sus implicaciones. En el caso de la caracterización del riesgo social se observa una naturalización de imágenes culturales, a través de las cuales ciertas conductas individuales son puestas en tela de juicio desde el ámbito experto. De apariencia neutral, estas representaciones sociales que instruyen el deber ser de las conductas y actitudes de los sujetos analizados, están fuertemente connotadas desde el punto de vista político y moral.

El lenguaje del riesgo, en el contexto de las condiciones sociales de su producción, permite al discurso ideológico delimitar factores de riesgo en las actitudes y conductas individuales que inmunizarían a las personas y grupos sociales contra el fracaso, mostrando a aquellos un ideal comunitario de salud y éxito social (Luhmann, 1992). Quienes no llegan a superar determinados parámetros establecidos socialmente, tanto para la producción, como para el consumo y la reproducción social, son señalados -mediante el discurso del riesgo-como no capaces, por sí mismos, de alcanzar 
ciertas metas, que no se sostienen sino políticamente. De esta forma, se dibuja un mercado de bienes sociales, en el que el individuo compite simbólica y materialmente en un contexto de desigualdad en el que algunos ganan y otros quedan relegados o excluidos.

Mediante el concepto de riesgo social se van, de esta forma, legitimando ciertas prácticas tecnocráticas en el arbitraje y manejo de los asuntos sociales que activarían o no los sistemas de protección social hacia la invalidez que, en estos términos, pasa de ser simplemente física a ser social. Los sistemas expertos desarrollan cada vez con mayor pericia un sistema de indicadores, que señalan como factores de riesgo para el ejercicio de la convivencia y los roles sociales, que los sujetos están llamados a desempeñar.

Sin embargo, cabría preguntarse, ¿constituyen estos factores, medidas universal -o al menos localmente- válidas para la descripción de fenómenos o situaciones sociales caracterizadas desde el riesgo social?

El mayor o menor nivel de protocolización de estos dispositivos de control social revela el grado de consenso que estos tipos de caracterización social generan entre los sistemas especializados. Las prácticas disciplinarias que se legitiman y se activan desde el trabajo técnico-como la medicina, por ejemplo-, han desarrollado un sistema de códigos expertos mediante los cuales se identifican y enumeran un conjunto de elementos para detectar y describir un cuadro clínico.

Durante mucho tiempo, el diagnóstico clínico se desarrolló asociado a la idea de peligro que, al no cumplirse, derivaba en fracaso de la predicción. Mediante el concepto de riesgo, las disciplinas técnicas desarrollan un sistema de protecciones hacia sus enunciados predictivos que no están sustentados sobre la certidumbre sino sobre la hipótesis de su probabilidad de ocurrencia (Castel, 1986; Mitjavila, 2002).

La utilización de un instrumental tan versátil y sofisticado en relación a la descripción, diagnóstico y predicción de comportamientos individuales y sociales -empleado sobre todo en poblaciones precariamente incluidas-genera entre los expertos tanta oposición como seducción. Pero, como dice Mitjavila, "ninguno de los dispositivos biopolíticos hasta ahora conocidos existe en sí mismo: el riesgo es pura forma y no contenido; 
permanece abierto, disponible para circular ampliamente en el espacio social y ofrecer así un soporte para transportar múltiples proyectos y significados sociales" (Mitjavila 2002: 143).

\section{Descripción y evaluación: el peso del diagnóstico en las trayectorias institucionales de los niños y adolescentes uruguayos}

Como ya se señaló, cuando un niño o adolescente ingresa a la institución pública responsable de la asistencia social a través del dispositivo de captación judicial, viene precedido de un dictamen experto que da cuenta de una serie de factores psicológicos, económicos y sociales que lo colocan en situación de riesgo social. La situación mediante la que el sujeto es individualizado se configura a partir de una detección precoz y preventiva que se dibuja a partir de la identificación "probabilística y abstracta de riesgos" (Castel, 1986).

Las estructuras y dispositivos institucionales de control que se ponen en juego mediante la prevención dan cuenta de lo que Castel llama una "nueva modalidad de vigilancia" cuyo objetivo es "anticipar e impedir la emergencia de un suceso no deseable" (Castel 1986: 230).

El sujeto es pues, precedido de un conjunto de información que anticipa su conducta futura, predecible, para los sistemas expertos. Para esta prematura detección de futuros conflictos, dirá Castel, no hace falta la presencia de individuos concretos. El diagnóstico habla por ellos. Las lecturas expertas acumulan dictámenes técnicos que constituyen "historiales subterráneos" que anticipan la conducta de los sujetos en la "científica" fórmula de la prevención de futuros daños.

El diagnóstico anuncia y determina los trayectos individuales en una suerte de profecía que se auto-confirma mediante el recorrido de los individuos por instituciones que no los acogen, defienden o amparan sino que los ratifican en la estigmatización y segregación social previas a su ingreso. Al final del recorrido institucional se comprueba lo que ya se sabía: un individuo caracterizado en función de determinados factores de riesgo, identificado en su condición de vulnerabilidad, con un recorrido errático por una institución que lo confina al desamparo afectivo, se aleja de la 
institución de asistencia a la infancia y la adolescencia cuando cumple su "mayoría de edad" en condiciones similares o peores a las de su ingreso y es captado por nuevas instituciones de vigilancia que se sostienen en versiones ideológicas del control similares a las que ya transitara durante sus primeros años de vida.

En esta política preventiva desaparece el sujeto que queda subsumido en "correlaciones estadísticas de elementos heterogéneos" a través de los cuales se "descompone al sujeto concreto de la intervención” que se reconstruye a partir de la mirada experta en la historia "clínica" de sucesivos diagnósticos que enumeran y describen su historia de vida por medio de un complejo cálculo de probabilidades (CasteL, 1986: 231-232).

Este saber sobre el otro opera a través de un proceso de racionalización acerca de diferentes causalidades sociales -como el abandono, el maltrato, la pérdida de la capacidad de juicio de lo que es correcto e incorrecto, el conflicto de ciertas conductas con las disposiciones legales- que tiene implicaciones políticas.

Se trata de estereotipias racionalizadas y pragmáticas que operan en la actividad diagnóstica -tanto a nivel judicial como de política social- a través de una discriminación de factores de riesgo que no tienen como objetivo asistir al sujeto en su vulnerabilidad sino imprimirle una marca para la elaboración de un perfil de predisposición a la conducta socialmente reprobable o -como bien lo describe el diagnóstico psiquiátrico en toda su dureza sancionatoria- "antisocial”.

Esta señal, técnicamente impresa, acompaña al sujeto durante toda la trayectoria institucional confirmándose en cada instancia de evaluación diagnóstica, dispositivo que se activa automáticamente en cada episodio de la vida del sujeto que requiera de la intervención experta.

La mirada técnica "localiza" al sujeto, le pone una marca que no contribuye a aumentar su reflexividad -aspiración de los analistas del riesgo- ni lo acompaña en el proceso de autodescubrimiento, ni en la adaptación al hogar que lo recibe o en las distintas instancias de conflicto que supone la convivencia. La mirada del otro -portador del saber- reaparece en la biografía del sujeto cuando es requerida a partir de un desorden, una explosión, un "pasaje al acto" que requiere nuevamente de un diagnóstico y un pronóstico de su conducta futura. 
Volviendo al planteo de Giddens respecto al proceso de individuación que trae consigo la reflexividad del yo, el autor hablaba de una construcción de una trayectoria vital que podría revisarse a través de una sucesión de "momentos" que forman parte de un itinerario reflejamente activado de autorrealización. La pregunta que surge es ¿quién selecciona estos momentos significativos? Tanto a nivel judicial como en el contexto institucional de la protección social, la "selección" de estos momentos significativos en la vida de los niños y los adolescentes queda en manos del sistema especializado, que efectúa fotografías instantáneas donde establece el diagnóstico de situaciones complejas por las que atraviesa el sujeto en su crecimiento y desarrollo personal.

¿Corresponde a la administración de las instituciones la determinación de las intervenciones técnicas en la vida de los sujetos y al observador la ilación de estas situaciones de crisis?

¿Qué queda en pie de este proceso auto-reflexivo en condiciones de institucionalización? ¿Qué ocurre con el acompañamiento terapéutico de estos procesos de desamparo que se han tipificado como "riesgosos" desde el punto de vista social? La información empírica parece indicar que la mirada técnica "no ve" más allá del síntoma.

\section{Consideraciones finales}

El informe diagnóstico se presenta como una pieza de producción experta, de carácter afirmativo que informa, mediante la descripción y el análisis de una situación, una modalidad de ser y de relacionarse de un sujeto y su grupo familiar que es considerada en función de su riesgo social.

Ante la interrogante acerca de la conceptualización de riesgo con la que operan los expertos en el ámbito socio-jurídico, las indagaciones realizadas en el marco de la investigación que da origen a este trabajo, evidencian una forma de argumentación técnica que parece apoyarse en las bases argumentales de la modernidad reflexiva.

Desde el supuesto de que la individuación -como proceso intrínseco de la modernidad-construye subjetividades reflexivas y autocríticas, que asumen responsablemente las consecuencias de las decisiones adoptadas en sus trayectorias de vida, se dibuja una matriz diagnóstica que puede reconocerse detrás de los 
pareceres técnicos que se aplican a los sujetos que llegan a la institución encargada de su protección y asistencia, provenientes de un arbitraje realizado en el sistema de justicia.

El cuerpo de expertos retoma el lenguaje del riesgo a partir del supuesto de la construcción auto-reflexiva de la biografía de sujetos que disponen de la información necesaria para asumir responsablemente las consecuencias de sus decisiones y dar cuenta de sus conductas de forma racional. Se concibe entonces, un ser humano, que en palabras de Beck "es capaz de escoger, decidir y crear, que aspira a ser autor de su propia vida, creador de una identidad individual" (Beck 2001: 234).

De esta manera, continúa Beck, los hechos de la vida no se atribuyen a "causas ajenas", "sino a aspectos del individuo (decisiones, indecisiones, omisiones, capacidades, incapacidades, logros, concesiones, derrotas)" (Beck 2001: 238).

Se borran así, vulnerabilidades socialmente inscriptas en una sociedad de clases atribuyendo a los sujetos individuales y a grupos familiares más frágiles y desestabilizados la responsabilidad de conductas que atentan contra una concepción aséptica de salud pública y de orden social.

De esta forma, se estigmatiza la pobreza que es tratada en función de la irresponsabilidad social de los sectores excluidos, a los que se culpabiliza de su situación de minoridad social.

Mediante un sofisticado mecanismo de atribución de responsabilidades, el informe diagnóstico descontextualiza el análisis de los conflictos sociales que surgen en una sociedad excluyente, cuya desigualdad no es posible resolver desde lógicas individuales y voluntarias.

De la información empírica recogida en la investigación de referencia surge que la exposición de los asuntos sociales ante la justicia implica una agudización de la tendencia hacia la individualización de los riesgos y la privatización de los problemas sociales. Bajo la lupa del peritaje técnico, los conflictos sociales desaparecen y se transforman en asuntos individuales frente a los que las personas no han logrado una solución satisfactoria.

De esta manera es posible identificar una forma de control social, que se asienta en un diagnóstico especializado que "adquiere fuerza de verdad en la clasificación de los sujetos" (Costa y Gagliano; 2000: 77) a quienes no atiende en su desamparo, sino mediante la denuncia 
de su "incapacidad" de resolver los asuntos más "nimios" de su biografía.

Con estos elementos ¿podría ser posible pensar estas prácticas diagnósticas en el marco de un mundo que si bien se presenta "desbocado", como afirma Beck (2001), se observa fundamentalmente "deshonrado", en el sentido de la afirmación de Autés (2005: 28), cuando señala que asistimos al "deshonor de una sociedad que no logra ya afrontar cierta cantidad de responsabilidades" y que obliga a los sujetos a cargar "con el peso de sí mismos y de la conducción de sus vidas, con recursos muy escasos" (Castel; 2005:86)?

Como argumenta Bauman, contraponiendo la perspectiva de los analistas del riesgo y su visión de la responsabilidad colectiva en la privatización de los asuntos sociales, "El poder de licuefacción se ha desplazado del «sistema» a la «sociedad», de la «política» a las «políticas de la vida» o ha descendido desde el «macronivel» al «micronivel» de la cohabitación social. Como resultado, la nuestra es una visión privatizada de la modernidad, en la que el peso de la construcción de pautas y la responsabilidad del fracaso caen primordialmente sobre los hombros del individuo" (Bauman, 2002: 13).

En definitiva, la vida propia que enuncia Beck (2001) -y que brega por la realización y el triunfo individual- supone que el fracaso también es propio.

\section{Referencias bibliográficas}

AUTÉS, M. (2005) “Tres formas de desligadura”, en Karsz, S. (coord.) La exclusión: bordeando sus fronteras. Definiciones y matices. Gedisa, Barcelona.

BAUMAN, Z. (2002) Modernidad líquida. Fondo de Cultura Económica, Buenos Aires.

BECK, U. (1994) "La reinvención de la política: hacia una teoría de la modernidad reflexiva “, en Beck, U.; Giddens, A y Lash, S. Modernización reflexiva. Política, tradición y estética en el orden social moderno. Alianza Universidad. Madrid.

(1998) La sociedad del riesgo: hacia una nueva modernidad. Paidós. Barcelona.

(2001) "Vivir nuestra propia vida en un mundo desbocado: individuación, globalización y política", en Giddens, A. y Hutton, W. (eds) En el límite. La 
vida en el capitalismo global. Kriterios TusQuets. Editores. Barcelona.

CASTEL, R. (1986) "De la peligrosidad al riesgo", en Materiales de Sociología crítica. La Piqueta, Madrid.

(2004) La inseguridad social ¿Qué es estar protegido? Manantial. Buenos Aires.

(2005) “Encuadre de la exclusión”, en Karsz, S. (coord.) La exclusión: bordeando sus fronteras. Definiciones y matices. Gedisa, Barcelona.

COSTA, M.; GAGLIANO, R. (2000) "Las infancias de la minoridad", en Duschantzky, S. (comp.) Tutelados y asistidos. Programas sociales, políticas públicas y subjetividad. Paidós, Buenos Aires.

GIDDENS, A. (1994) Consecuencias de la modernidad. Alianza Editorial, Madrid.

(1995) Modernidad e identidad del yo. Península. Barcelona.

LUHMANN. N. (1992) Sociología del riesgo. Universidad Latinoamericana, Universidad de Guadalajara, México.

MITJAVILA, M. (2002) "O risco como recurso para a atribragem social". Tempo Social, Revista de Sociologia, UPS, São Paulo, 14 (2), octubro.

SVAMPA, M. (2000) (editora) Desde abajo. La transformación de las identidades sociales. Universidad Nacional de General Sarmiento. Editorial Biblos. Buenos Aires. 